PROCEEDINGS OF THE

AMERICAN MATHEMATICAL SOCIETY

Volume 131, Number 4, Pages $1177-1180$

S 0002-9939(02)06638-8

Article electronically published on November 4, 2002

\title{
THE RANK OF HANKEL OPERATORS ON HARMONIC BERGMAN SPACES
}

\author{
LOVA ZAKARIASY
}

(Communicated by Joseph A. Ball)

\begin{abstract}
We show that on the harmonic Bergman spaces, the Hankel operators with nonconstant harmonic symbol cannot be of finite rank.
\end{abstract}

\section{INTRODUCTION}

Let $D$ denote the open unit disk in the complex plane $\mathbb{C}$ and let $d A$ denote normalized Lebesgue measure on $D$. The harmonic Bergman space $L_{h}^{2}$ is the space of harmonic functions on $D$ which are in $L^{2}=L^{2}(D, d A)$.

The set $L_{h}^{2}$ with the inner product

$$
\langle u, v\rangle=\int_{D} u \bar{v} d A
$$

and orthonormal basis $\left\{1, \sqrt{2} z, \sqrt{2} \bar{z}, \ldots, \sqrt{n+1} z^{n}, \sqrt{n+1} \bar{z}^{n}, \ldots\right\}$ is a Hilbert space.

If $P$ is the orthogonal projection from $L^{2}$ onto $L_{h}^{2}$, the Hankel operator with symbol $f \in L^{2}$ is defined by

$$
H_{f}: L_{h}^{2} \rightarrow\left(L_{h}^{2}\right)^{\perp} \quad \text { such that } \quad H_{f}(u)=(I-P)(f u) .
$$

In this paper, we show that there are no Hankel operators with nonconstant harmonic symbols which are of finite rank. The finite rank Hankel operators on the Bergman space $L_{a}^{2}=L^{2} \cap \operatorname{Hol}(D)$ are characterized in [1]. On $L_{a}^{2}$ we define the big and the small Hankel operators respectively by

$$
H_{f}^{b i g}(\varphi)=\left(I-P_{a}\right)(f \varphi) \quad \text { and } \quad H_{f}^{s m}(\varphi)=Q_{a}(f \varphi)
$$

where $P_{a}$ and $Q_{a}$ are the projections from $L^{2}$ onto $L_{a}^{2}$ and $\overline{L_{a}^{2}}$ respectively. If $f$ is analytic, $H_{\bar{f}}^{b i g}$ is not of finite rank on $L_{a}^{2}$ unless $f$ is a constant function. On the other hand, the small Hankel operator $H \frac{s m}{f}$ is of finite rank on $L_{a}^{2}$ if and only if the symbol is a linear combination of the reproducing kernels and some of their derivatives (see [1]). Similar results have been shown for the intermediate Hankel operators on the Bergman space (see [3] ).

It is not surprising that linear combinations of reproducing kernels are always symbols of finite rank operators on the Bergman space. For $w \in D$, let $h_{w}$ be the

Received by the editors September 14, 2001 and, in revised form, November 11, 2001.

2000 Mathematics Subject Classification. Primary 47B35.

Key words and phrases. Hankel operators, harmonic Bergman spaces.

(C)2002 American Mathematical Society 
reproducing kernel in $w$ for the complex conjugate Bergman space $\overline{L_{a}^{2}}$ :

$$
h_{w}(z)=h(w, z)=(1-w \bar{z})^{-2} .
$$

Then $\left\langle\bar{g}, h_{w}\right\rangle=\bar{g}(w)$ for all $\bar{g} \in \overline{L_{a}^{2}}$ and, by differentiating under the integral, we see that for $h_{w}^{(k)}=\frac{\partial^{k}}{\partial w^{k}} h(w, z)$

$$
\bar{g}^{(k)}(w)=\frac{\partial^{k} \bar{g}}{\partial \bar{w}^{k}}(w)=\left\langle\bar{g}, h_{w}^{(k)}\right\rangle
$$

So, if $\varphi \in L_{a}^{2}$, and $\bar{g} \in \overline{L_{a}^{2}}$

$$
\begin{aligned}
\left\langle\bar{g}, H_{h_{w}^{(k)}}^{s m}(\varphi)\right\rangle & =\left\langle\bar{g}, Q_{a}\left(\varphi h_{w}^{(k)}\right)\right\rangle \\
& =(\overline{g \varphi})^{(k)}(w) \\
& =\sum_{j=0}^{k} C_{k}^{j} \bar{\varphi}^{(k-j)}(w)\left\langle\bar{g}, h_{w}^{(j)}\right\rangle .
\end{aligned}
$$

Thus $H_{h_{w}^{(k)}}^{s m}(\varphi)=\sum_{j=0}^{k} C_{k}^{j} \varphi^{(k-j)}(w) h_{w}^{(j)}$, so the range of the small Hankel operator with the symbol $h_{w}^{(k)}$ is spanned by the reproducing kernel $h_{w}$ and its $k$ first partial derivatives.

In the harmonic Bergman space, there is an analogy between the small Hankel operators and the Toeplitz operators $T_{f}: u \in L_{h}^{2} \rightarrow P(f u)$. In fact, $\overline{L_{h}^{2}}=L_{h}^{2}$ and so the analogue of $H_{f}^{s m}$ is $\Gamma_{f}^{s m}(u)=P(f u)$ which is the usual definition of a Toeplitz operator. In spite of this analogy, the argument above does not work here for the simple reason that the product of two harmonic functions is not harmonic. Furthermore, it is shown in [4 that the only compact Toeplitz operator with a harmonic symbol on the harmonic Bergman spaces is the zero operator. So, no Toeplitz operator with a non-zero harmonic symbol is of finite rank on $L_{h}^{2}$.

In the next section, we study Hankel operators with a monomial symbol, then show that the Hankel operators with analytic or antianalytic symbol are not of finite rank on the harmonic Bergman space. From this we conclude that there are no finite rank Hankel operators with nonconstant harmonic symbols. In this paper, we exclude the trivial case when the symbol is a constant function.

\section{RANK of Hankel operators}

Let $k \in \mathbb{Z}$. We say that the polynomial $p_{k}$ is quasihomogenous of degree $k$ if it can be written as

$$
p_{k}\left(r e^{i} \theta\right)=q(r) e^{i k \theta}
$$

where $q(r)$ is a real polynomial and $r$ and $\theta$ are the polar coordinates of the complex variable $z$.

We can immediately see that two quasihomogenous polynomials of different degrees are orthogonal in $L_{h}^{2}$. The following lemma uses the fact that the image of a monomial by a Hankel operator with a monomial symbol is a quasihomogenous polynomial.

Lemma 2.1. Let $n$ be a positive integer. Then:

(a) $H_{\bar{z}^{n}}\left(z^{k}\right) \perp H_{\bar{z}^{n}}\left(z^{j}\right) \quad$ if $j \neq k$, 
(b) $\left\|H_{\bar{z}^{n}}\left(z^{k}\right)\right\|= \begin{cases}\frac{k}{(n+1) \sqrt{k+n+1}} & \text { if } k \leq n, \\ \frac{n}{(k+1) \sqrt{k+n+1}} & \text { if } k \geq n .\end{cases}$

Proof. It is easy to calculate the projection of the monomials

$$
P\left(\bar{z}^{n} z^{k}\right)= \begin{cases}\frac{n-k+1}{n+1} \bar{z}^{n-k} & \text { if } k \leq n, \\ \frac{k-n+1}{k+1} z^{k-n} & \text { if } k \geq n .\end{cases}
$$

With this formula, notice that $H_{\bar{z}^{n}}\left(z^{k}\right)$ is a quasihomogenous polynomial of degree $k-n$. This proves (a). An immediate calculation gives the value of the $L^{2}$-norm of $H_{\bar{z}^{n}}\left(z^{k}\right)$ in (b).

Observing the degree of each quasihomogenous polynomial, we remark that $H_{\bar{z}^{n}}\left(z^{k}\right)$ is not orthogonal to $H_{\bar{z}^{m}}\left(z^{j}\right)$ if and only if $k-n$ equals $j-m$.

Proposition 2.2. Let $\varphi$ and $\psi$ be two functions in $L_{a}^{2}$. The Hankel operators $H_{\varphi}$ and $H_{\bar{\psi}}$ are not of finite rank on the harmonic Bergman space $L_{h}^{2}$.

Proof. Let us show that $H_{\varphi}$ is not of finite rank. The same proof works for an antianalytic symbol. Let $\varphi=\sum_{k=0}^{\infty} a_{k} z^{k}$. We may assume, without loss of generality, that $\varphi(0)=0$, because $H_{\varphi-\varphi(0)} \equiv H_{\varphi}$. We denote by $s_{0}$ the least index such that

$$
a_{1}=a_{2}=\ldots=a_{s_{0}-1}=0 \text { and } a_{s_{o}} \neq 0 .
$$

Fix $n$ :

$$
H_{\varphi}\left(\bar{z}^{n}\right)=\sum_{k=s_{0}}^{\infty} a_{k}(I-P)\left(z^{k} \bar{z}^{n}\right)=\sum_{k=s_{0}}^{\infty} a_{k} H_{\bar{z}^{n}}\left(z^{k}\right) .
$$

Hence $H_{\varphi}\left(\bar{z}^{n}\right)$ is the sum of quasihomogenous polynomials of degree $k-n$, where $k \geq s_{0}$. We need the following result, which is easy to check. If $m$ and $n$ are given, then

$$
\left\langle H_{\varphi}\left(\bar{z}^{n}\right), H_{\bar{z}^{m}}\left(z^{s_{0}}\right)\right\rangle= \begin{cases}a_{s_{0}}\left\|H_{\bar{z}^{n}}\left(z^{s_{0}}\right)\right\|^{2} & \text { if } m=n, \\ 0 & \text { if } m>n .\end{cases}
$$

Now, suppose that there exist scalars $\lambda_{1}, \lambda_{2}, \ldots, \lambda_{p}$, such that

$$
\sum_{n=1}^{p} \lambda_{n} H_{\varphi}\left(\bar{z}^{n}\right)=0
$$

From the previous remark

$$
0=\left\langle\sum_{n=1}^{p} \lambda_{n} H_{\varphi}\left(\bar{z}^{n}\right), H_{\bar{z}^{p}}\left(z^{s_{0}}\right)\right\rangle=\lambda_{p} a_{s_{0}}\left\|H_{\bar{z}^{p}}\left(z^{s_{0}}\right)\right\|^{2},
$$

which implies $\lambda_{p}=0$. Continuing with the same method we find

$$
\lambda_{p}=\lambda_{p-1}=\ldots=\lambda_{1}=0,
$$

and this shows that the set $\left\{H_{\varphi}\left(\bar{z}^{n}\right)\right\}_{n=1}^{p}$ is linearly independent for all $p>0$. Finally, $H_{\varphi}$ is not of finite rank if $\varphi \in L_{a}^{2}$.

Corollary 2.3. If $f \in L_{h}^{2}$, then the Hankel operator $H_{f}$ is not of finite rank on the harmonic Bergman space. 
Proof. Let $f \in L_{h}^{2}$. Hence, $f=\varphi+\bar{\psi}$, where $\varphi$ and $\psi$ are from $L_{a}^{2}$. For all functions $g \in L_{a}^{2}, H_{\varphi}(g)=0$ and $H_{\bar{\psi}}(\bar{g})=0$. So we have

$$
\left.H_{f}\right|_{L_{a}^{2}}=H_{\bar{\psi}} \quad \text { and }\left.\quad H_{f}\right|_{\overline{L_{a}^{2}}}=H_{\varphi} .
$$

But $\left.H_{f}\right|_{L_{a}^{2}}$ is of finite rank only if $\psi=0$, in which case $\varphi \neq 0$ and so $\left.H_{f}\right|_{\overline{L_{a}^{2}}}$ is not of finite rank. So, the Hankel operator $H_{f}$ cannot be of finite rank.

Notice that from Lemma 2.1, one can deduce very easily a result about the compacity on $L_{h}^{2}$ of Hankel operators, when the symbol is continuous in the closed disk.

Proposition 2.4. The Hankel operator $H_{\bar{z}^{n}}$ is a Hilbert-Schmidt operator, so it is compact on the harmonic Bergman space $L_{h}^{2}$.

Proof. By definition, $H_{\bar{z}^{n}}$ is a Hilbert-Schmidt operator if $\operatorname{Tr}\left(H_{\bar{z}^{n}}^{*} H_{\bar{z}^{n}}\right)<\infty$. This is easy to prove, using the formulas from Lemma 2.1. We apply $H_{z^{n}}^{*} H_{\bar{z}^{n}}$ to the elements of the orthonormal basis of $L_{h}^{2}$ and we have

$$
\begin{aligned}
\operatorname{Tr}\left(H_{\bar{z}^{n}}^{*} H_{\bar{z}^{n}}\right) & =\sum_{k=0}^{\infty}(k+1)\left\|H_{\bar{z}^{n}}\left(z^{k}\right)\right\|^{2} \\
& =\sum_{k=0}^{n} \frac{k^{2}(k+1)}{(k+n+1)(n+1)^{2}}+\sum_{k=n+1}^{\infty} \frac{n^{2}}{(k+n+1)(k+1)} .
\end{aligned}
$$

It is clear that this sum is convergent. Thus, $H_{\bar{z}^{n}}$ is a Hilbert-Schmidt operator, hence compact.

Proceeding in the same way, we see that $H_{z^{n}}$ is also compact.

Corollary 2.5. Any Hankel operator with a continuous symbol in the closed disk is compact on the harmonic Bergman space $L_{h}^{2}$.

This corollary is due to the fact that polynomials are dense in $C(\bar{D})$. So, the compacity of $H_{z^{n}}$ and $H_{z^{n}}$ easily implies the compacity of Hankel operators with continuous symbol. This property has been proved in [2] using another method.

\section{REFERENCES}

[1] N. Das, The kernel of a Hankel operator on the Bergman space, Bull. London Math. Soc. 31 (1999), 75-80. MR 99j:47034

[2] M. Jovovic, Compact Hankel operators on Harmonic Bergman spaces, Integral Equations Operator Theory, Vol. 22, 1995, 295-304. MR 96d:47031

[3] E. Strouse, Finite rank intermediate Hankel operators, Arch. Math. (Basel), Vol. 67, 1996, 142-149. MR 97i:47047

[4] Z. Wu, Operators on harmonic Bergman spaces, Integral Equations Operator Theory, Vol. 24, 1996, 352-371. MR 97c:47028

Department of Mathematics, University of Bordeaux I, 351, Cours de la Liberation, 33045 Talence cedex, France

E-mail address: lova.zakariasy@math.u-bordeaux.fr 